\title{
MATRIX REARRANGEMENT INEQUALITIES REVISITED
}

\author{
VICTORIA M. CHAYES
}

Abstract. Let $\|X\|_{p}=\operatorname{Tr}\left[\left(X^{*} X\right)^{p / 2}\right]^{1 / p}$ denote the $p$-Schatten norm of a matrix $X \in M_{n \times n}(\mathbb{C})$, and $\sigma(X)$ the singular values with $\uparrow \downarrow$ indicating its increasing or decreasing rearrangements. We wish to examine inequalities between $\|A+B\|_{p}^{p}+\|A-B\|_{p}^{p},\left\|\sigma_{\downarrow}(A)+\sigma_{\downarrow}(B)\right\|_{p}^{p}+\| \sigma_{\downarrow}(A)-$ $\sigma_{\downarrow}(B) \|_{p}^{p}$, and $\left\|\sigma_{\uparrow}(A)+\sigma_{\downarrow}(B)\right\|_{p}^{p}+\left\|\sigma_{\uparrow}(A)-\sigma_{\downarrow}(B)\right\|_{p}^{p}$ for various values of $1 \leqslant p<\infty$. It was conjectured in [6] that a universal inequality $\left\|\sigma_{\downarrow}(A)+\sigma_{\downarrow}(B)\right\|_{p}^{p}+\left\|\sigma_{\downarrow}(A)-\sigma_{\downarrow}(B)\right\|_{p}^{p} \leqslant$ $\|A+B\|_{p}^{p}+\|A-B\|_{p}^{p} \leqslant\left\|\sigma_{\uparrow}(A)+\sigma_{\downarrow}(B)\right\|_{p}^{p}+\left\|\sigma_{\uparrow}(A)-\sigma_{\downarrow}(B)\right\|_{p}^{p}$ might hold for $1 \leqslant p \leqslant 2$ and reverse at $p \geqslant 2$, potentially providing a stronger inequality to the generalization of Hanner's Inequality to complex matrices $\|A+B\|_{p}^{p}+\|A-B\|_{p}^{p} \geqslant\left(\|A\|_{p}+\|B\|_{p}\right)^{p}+\||| A\|_{p}-\left.\|B\|_{p}\right|^{p}$. We extend some of the cases in which the inequalities of [6] hold, but offer counterexamples to any general rearrangement inequality holding. We simplify the original proofs of [6] with the technique of majorization. This also allows us to characterize the equality cases of all of the inequalities considered. We also address the commuting, unitary, and $\{A, B\}=0$ cases directly, and expand on the role of the anticommutator. In doing so, we extend Hanner's Inequality for self-adjoint matrices to the $\{A, B\}=0$ case for all ranges of $p$.

Mathematics subject classification (2010): 15A42.

Keywords and phrases: Matrix inequality, Hanner's inequality, singular value inequalities, $p$-Schatten norm, majorization.

\section{REFERENCES}

[1] F. J. Almgren JR, E. H. Lieb, Symmetric decreasing rearrangement is sometimes continuous Journal of the American Mathematical Society, pp. 683-773 (1989).

[2] T. ANDo, Majorization, doubly stochastic matrices, and comparison of eigenvalues, Linear Algebra and its Applications 118, 163-248 (1989), https : //doi .org/10.1016/0024-3795 (89) 90580-6, http://www.sciencedirect.com/science/article/pii/0024379589905806.

[3] K. BALL, E. A. CARLEN, E. H. LIEB, Sharp uniform convexity and smoothness inequalities for trace norms, Inventiones mathematicae 115 (1), 463-482 (1994), https://doi.org/10.1007/BF01231769.

[4] J. C. Bourin, E. Y. LEE, Clarkson-McCarthy inequalities with unitary and isometry orbits, Linear Algebra and its Applications 601, 170-179 (2020), https://doi.org/10.1016/j.laa.2020.04.019, http://www.sciencedirect.com/science/article/pii/S0024379520302135.

[5] A. Burchard, Cases of equality in the riesz rearrangement inequality, Annals of Mathematics 143 (3), 499-527 (1996). http://www.jstor.org/stable/2118534.

[6] E. Carlen, E. H. Lieb, Some matrix rearrangement inequalities, Annali di Matematica Pura ed Applicata 185 (5), S315-S324 (2006), https://doi .org/10.1007/s10231-004-0147-z.

[7] K. FAN, Maximum properties and inequalities for the eigenvalues of completely continuous operators, Proceedings of the National Academy of Sciences of the United States of America 37 (11), 760-766 (1951), 10.1073/pnas.37.11.760, https://www.ncbi.nlm.nih.gov/pubmed/16578416.

[8] I. M. GEL'FAND, M. A. NAIMARK, The relation between the unitary representations of the complex unimodular group and its unitary subgroup, Izv. Akad. Nauk SSSR Ser. Mat. 14 (3), 239-260 (1950).

[9] G. H. HARdY, J. E. LitTlewood, G. Pólya, Some simple inequalities satisfied by convex functions, Messenger Math. 58, 145-152 (1929), https://ci.nii.ac.jp/naid/10009422169/en/. 
[10] G. H. Hardy, G. Pólya, Inequalities, Cambridge: Cambridge University Press (1934), Bibliography: p. 300-314.

[11] F. HIAI, Equality cases in matrix norm inequalities of golden-thompson type, Linear and Multilinear Algebra 36 (4), 239-249 (1994), doi : 10.1080/03081089408818297, https://doi.org/10.1080/03081089408818297.

[12] F. HiAi, D. Petz, Introduction To Matrix Analysis And Applications, 1 edn., chap. 6, pp. 227-271, Springer International Publishing, Cham (2014).

[13] A. HoRn, On the singular values of a product of completely continuous operators, Proceedings of the National Academy of Sciences of the United States of America 36 (7), 374 (1950).

[14] A. W. Marshall, I. Olkin, B. C. ARnold, Inequalities: Theory of Majorization and Its Applications, 2 edn., Springer, New York (2011).

[15] C. MCCARTHY, c.p cp, Isr. J. Math. 5, 249-271 (1967).

[16] N. TomcZAK-JAEgermann, The moduli of smoothness and convexity and the Rademacher averages of the trace classes $S_{p}(1 \leqslant p \leqslant \infty)^{*}$, Studia Mathematica 50 (2), 163-182 (1974), http://eudml.org/doc/217886.

[17] M. Tомić, Théoreme de gauss relatif au centre de gravité et son application, Bull. Soc. Math. Phys. Serbie 1, 31-40 (1949).

[18] H. WEYL, Inequalities between two types of eigenvalues of a linear transformation, Proceedings of the National Academy of Sciences of the United States of America 35 (7), 408-411 (1949). 